Original research paper

\title{
Protective Effect of Lactoferrin against Chromium Induced Adverse Renal Changes in Rats: Oxidative Stress Theory
}

\author{
1,"Mohammed H. Hassan, ${ }^{2}$ Dorreia Abd-Alla Mohamed Zaghloul, \\ ${ }^{3}$ Marwa Ahmed Mahmoud, ${ }^{4}$ Zamzam Nasrallah Abdel-Moaty and ${ }^{5}$ Rana Toghan \\ ${ }^{I}$ Department of Medical Biochemistry, Faculty of Medicine, South Valley University, Qena, Egypt \\ ${ }^{2}$ Department of Human Anatomy and Embryology, Faculty of Medicine, Assiut University, Assiut, Egypt \\ ${ }^{3}$ Department of Medical Physiology, Faculty of Medicine, Sohag University, Sohag, Egypt \\ ${ }^{4}$ Department of Human Anatomy and Embryology, Faculty of Medicine, South Valley University, Qena 83523, Egypt \\ ${ }^{5}$ Department of Medical Physiology, Faculty of Medicine, South Valley University, Qena 83523, Egypt
}

Article history

Received: 22-02-2021

Revised: 10-04-2021

Accepted: 15-04-2021

Corresponding Author:

Mohammed H. Hassan

Department of Medical

Biochemistry, Faculty of

Medicine, South Valley

University, Qena, Egypt

Email:

Mohammedhosnyhassaan@yahoo.com mohammedhosnyhassaan@med.svu.edu.eg

\begin{abstract}
Among the toxic metals, chromium $(\mathrm{Cr})$ which is a naturally occurring heavy metal commonly enters the environment through the effluents from various industries. Kidney disease is often cited as an adverse effect of chromium. We aimed to assess chromium-induced pathophysiological adverse renal damage and determine the potential protective effects of co-therapy with Lactoferrin (LF) in male albino rats. Forty male albino Wister rats were used in this study, allocated into 4 groups $(\mathrm{n}=10$ each). Group-I (control group) and received 1\% DMSO; group-II (LF group); group-III [Potassium Dichromate (PDC) group] and group-IV (PDC+LF group). Biochemical measurements of renal function (urea and creatinine), serum glucose and Total Antioxidant capacity (TAO) were performed using colorimetric methods. Renal tissue samples were used for histopathological examinations using light and transmission electron microscopic photomicrographs. There were significantly higher serum urea, creatinine and glucose with significantly lower TAO among PDC group $(69.4 \mathrm{mg} / \mathrm{dl} \pm 44.15,0.566 \mathrm{mg} / \mathrm{dl} \pm 0.13,140.8 \mathrm{mg} / \mathrm{dl} \pm 50.27$ and $6.22 \pm 2.09 \mathrm{mmol} / \mathrm{ml}$ ) compared to both the control group (45.2 \pm 7.44 , $0.492 \pm 0.07,113 \pm 15.23$ and $8 \pm .0 .84)$ and LF group (38.8 \pm 7.49 , $0.402 \pm 0.03,110.8 \pm 27.95$ and $8.02 \pm 1.05), \mathrm{p}<0.05$ for all. In addition, significanly lower serum urea and higher TAO among PDC+LF group (45.2 \pm 4.02 and $7.94 \pm 1.53$ ) were evidenent compared to PDC group, $\mathrm{p}<0.05$ for both. There were histological similarities in both control and LF groups with significant structural damage of the kidneys of PDC group and significant improvment of such damage in PDC+LF treated group. Lactoferrin could have a renoprotective effect against drug induced nephrotoxicity via its antioxidant property.
\end{abstract}

Keywords: Potassium Dichromate, Nephrotoxicity, Lactoferrrin, Oxidative Stress

\section{Introduction}

Owing to their toxicological and physiological effects on the environment, heavy metals have become synonymous with industrial pollution. Heavy metals can be absorbed orally, by inhalation, or through the skin (Al-Othman et al., 2012). Among the toxic metals, Chromium $(\mathrm{Cr})$ which is a naturally occurring heavy metal used for chrome plating, in the manufacture of dyes, steel, alloys and pigments, leather tanning and wood preserving. Via the effluent from these industries, chromium usually enters the environment. It is a significant cause of environmental contamination once it is released into the soil and water (El-Saad et al., 2010; Mishra and Bharagava, 2016). The most prevalent and stable forms of $\mathrm{Cr}$ in the environment are hexavalent 
Chromium [Cr (IV)] and the most toxic form of $\mathrm{Cr}$ (IV) is potassium dichromate (Wu et al., 2012; Mehany et al., 2013). Potassium Dichromate (PDC, $\mathrm{K}_{2} \mathrm{Cr}_{2} \mathrm{O}_{7}$ ) is a crystalline ionic solid, with a bright red-orange hue, most widely used as an oxidising agent in different laboratory and industrial applications. PDC is used for washing, leather, photography and building applicaations (Navya et al., 2018). It is a strong oxidising agent showing a marked affinity to form many complexes with various biological ligands, including nucleic acids, when reduced to trivalent chromium $\left(\mathrm{Cr}^{+3}\right)$ by numerous cell metabolites (Calvello et al., 2016).

By inhibiting antioxidant enzymes and binding to antioxidant elements such as Glutathione (GSH), chromium has the ability to alter cellular functions, contributing to oxidative stress (Kart et al., 2016). Ingestion, dermal contact and inhalation are the most common exposure routes for chromium (Sun et al., 2015).

As an adverse consequence of chromium, kidney failure is often cited. In comparison to the lack of evidence for chromium-induced chronic renal disease, massive hexavalent chromium toxicity appears to cause Acute Tubular Necrosis (ATN) (Teklay, 2016). In the proximal convoluted tubule, chromium compounds are selectively accumulated where, following parenteral administration, they cause acute tubular necrosis in large doses. There is reason to assume, along with the discovery of tubular proteinuria in chromium workers that chromium contributes to the production of chronic renal failure (Teklay, 2016).

Lactoferrin (LF) is an $80 \mathrm{kDa}$ member of the iron binding glycoprotein transferrin family that was first found in human milk (Yao et al., 2013). The human kidney production of LF has been identified (Åbrink et al., 2000). LF is expressed and secreted in the collecting tubules and it may be reabsorbed in the distal part of the tubules. In a highly ordered way, the kidney produces LF and only a small fraction of this protein is secreted into the urine. LF is also believed to have essential roles in both the urinary tract's immune response and in the metabolism of iron in general (Adlerova et al., 2008). The aim of the current study was to assess the biochemical and histopathological adverse effects of PDC on male rat kidneys. Also, to determine the potential protective effect of co-therapy with LF on adverse renal changes caused by PDC.

\section{Materials and Methods}

\section{Chemicals}

Potassium Dichromate (PDC) and lactoferrin were purchased from Sigma-Aldrich Company (St. Louis, MO, USA). All used reagents were of analytical grade and highest purity. The CAS numbers were 777-50-9 and 936541-36-5, respectively.

\section{Animals and Treatment}

In this study, 40 male albino Wister rats, 3-6 months of age, weighted $200 \pm 50 \mathrm{~g}$ were used. They were kept under a well-regulated light and dark (12 h: $12 \mathrm{~h}$ ) schedule at $22-25^{\circ} \mathrm{C}$. The animal had free access to tap water and treated according to the guidelines of the Animal House of South Valley University-Qena, where standard commercial pellets were used for feeding and water $a d$ libitum. All experimental protocols were performed in accordance with the local institutional guidelines and approved by the Animal Ethical Committee, South Valley University-Qena, Egypt.

The included rats were divided randomly into 4 groups $(\mathrm{n}=10$ each). All treatments were administered single dose daily. All chemicals were dissolved in $1 \%$ Dimethyl Sulfoxide (DMSO) and rats were treated daily for 14 days as follow (Fig. 1):

- Group-I (control group): Received 1\% DMSO orally.

- Group-II (LF group): Received lactoferrin (300 $\mathrm{mg} / \mathrm{kg}$ ) orally (Kimoto et al., 2013)

- Group-III (PDC group): Received potassium dichromate $(15 \mathrm{mg} / \mathrm{kg})$ Subcutaneous injection (Hegazy et al., 2016)

- Group-IV (PDC+LF): Received potassium dichromate $(15 \mathrm{mg} / \mathrm{kg})$ subcutaneous injection and lactoferrin $(300 \mathrm{mg} / \mathrm{kg})$ orally

\section{Sample Collection}

The animals were starved overnight at the end of the experimental phase ( 2 weeks) and anaesthetized using diethyl ether inhalation; then, at the time of scarification, the blood samples were collected from the retro-orbital veins into plain tubes and centrifuged for $10 \mathrm{~min}$ at $1000 \mathrm{~g}$ and the separated sera were split into aliquots in $1 \mathrm{~mL}$ cryotubes and preserved at $-80^{\circ} \mathrm{C}$ until biochemical assays were carried out (Saleem et al., 2018; Hassan et al., 2020). The kidneys were dissected out immediately and cleaned of adhering tissues and were immersed in neutral buffered formalin $10 \%$ for histopathology examination.

\section{Biochemical Analysis}

Serum urea, creatinine, glucose and Total Antioxidant capacity (TAO) measurements were performed, by colorimetric method (Chem-7, Erba Diagnostics Mannheim $\mathrm{GmbH}$, Germany), using commercially available assay kits supplied by Spectrum Company, Egypt, for urea, creatinine and glucose, with catalog no. 318 001, 253001 and 253001 respectively. While, TAO kit was supplied by Biodiagnostics, Egypt (Saleem et al., 2018; Hassan et al., 2019; Saleem et al., 2020). 


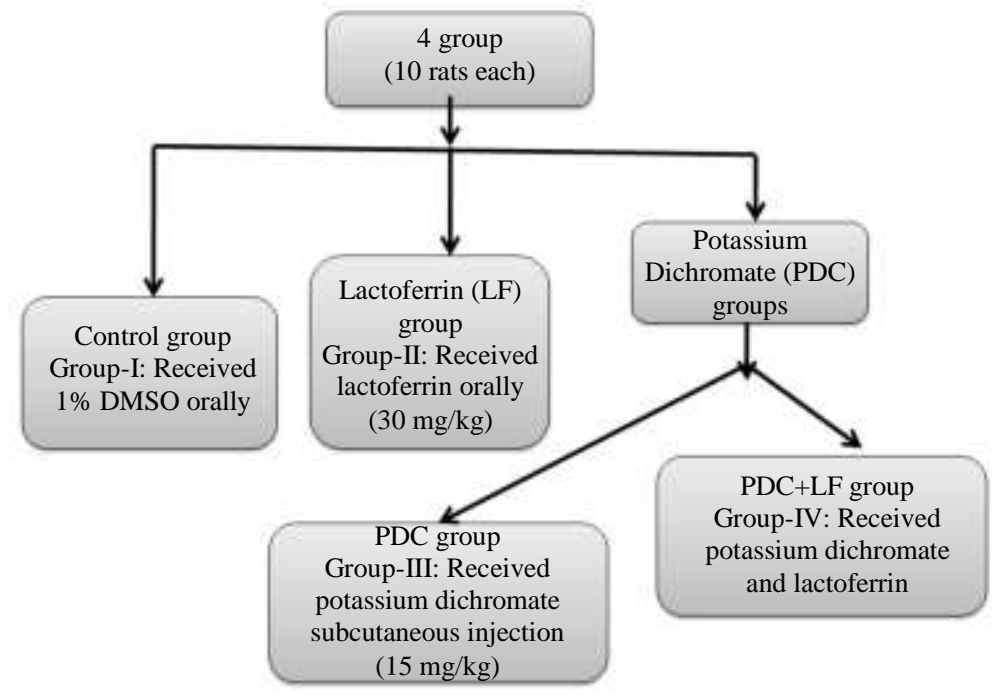

Fig. 1: Study design: Rats were divided randomly into 4 groups of 10 rats each; Abbreviations: DMSO: Dimethyl Sulfoxide; LF: Lactoferrin; PDC: Potassium Dichromate

\section{Histopathological Assessments}

Using traditional paraffin-embedding methods, processing of the fixed renal tissues was carried out. Using a microtome, five $\mu \mathrm{m}$ thick parts were obtained from prepared paraffin blocks. Then, hematoxylin and eosin staining stained these parts (Gabe, 1976; Gamble, 2008), for examination by light microscope. Additionally, histological technique for semi-thin and ultrathin renal tissue sections were performed (Ayache et al., 2010), where semi thin sectioning at $1 \mu \mathrm{m}$ renal specimens were trimmed with a razor blade and stained with $2 \%$ aqueous toluidine blue then dried on a hot plate at $40^{\circ} \mathrm{C}$ and were examined by light microscope. While, ultrathin sectioning at $50 \mathrm{~nm}$ were recommended on cooper grids and were examined by a transmission JEOLJEM-100CX II electron microscope and were photographed.

\section{Statistical Analysis}

The statistical analyses were carried out using SPSS version 22.0 (IBM, Armonk, NY, USA). Data with normal distribution (according to Kolmogoroy-Smimov test) were analyzed by one-way Analysis Of Variance (ANOVA) followed by Turkey's HSD post hoc test for comparison between multiple quantitative variables and were expressed as mean \pm SD. Statistical significance was considered when $\mathrm{p}<0.05$.

\section{Results}

\section{Serum Biochemical Assessments and Antioxidant Status of Various Study Groups}

Regarding to the biochemical evaluation of the kidney function among the study groups, there was statistically significant higher mean $\pm \mathrm{SD}$ blood urea $(\mathrm{mg} / \mathrm{dl})$ and serum creatinine $(\mathrm{mg} / \mathrm{dl})$ among PDC-treated rats (group-III)
$(69.4 \pm 44.15$ and $0.566 \pm 0.13$ respectively) compared to both the control group (group-I) $(45.2 \pm 7.44$ and $0.492 \pm 0.07$ respectively) and LF only-treated rats (groupII) $(38.8 \pm 7.49$ and $0.402 \pm 0.03$ respectively), $\mathrm{p}<0.05$ for all. Additionally, there were no significant differences between control group and PDC+LF treated rats (groupIV), $\mathrm{p}>0.05$ for both, (Table1).

As regards serum random blood glucose $(\mathrm{mg} / \mathrm{dl})$, there was significantly higher mean levels \pm SD among PDCtreated rats (group-III) $(140.8 \pm 50.27)$ compared to both the control group (group-I) and LF only-treated rats (group-II) $(113 \pm 15.23$ and $110.8 \pm 27.95$, respectively), $\mathrm{p}<0.05$ for all. However, there was no significant difference in the serum glucose levels among control group compared to PDC+LF treated rats (group-IV), $\mathrm{p}>0.05$, (Table1).

As regard the antioxidant status of the study groups, there were significant lower mean $\pm \mathrm{SD}$ serum total antioxidant capacity $(\mathrm{mmol} / \mathrm{ml})$ among PDC-treated rats (group-III) $(6.22 \pm 2.09)$ compared to each of the control group (group-I), LF only-treated rats (group-II) and PDC+LF treated rats (group-IV) $(8 \pm .0 .84,8.02 \pm 1.05$ and $7.94 \pm 1.53$ respectively), $\mathrm{p}<0.05$ for all. However, there was no significant difference in the serum TAO levels among control group compared to PDC+LF treated rats (group-IV), $\mathrm{p}>0.05$, (Table1).

\section{Histopathological Findings of the Rats' Kidneys among the Various Study Groups}

Regarding to control group and lactoferrin treated rats (group-I and II) using light microscopy (H\&E and semithin sections toluidine blue), the histopathological renal examinations revealed normal renal parenchyma, normal appearance of glomerulus and mesangial cells, proximal convoluted tubule, distal convoluted tubules, Urinary (Bowman's) space and vascular pole (Fig. 2A1$\mathrm{A} 2$ and $3 \mathrm{~B} 1-\mathrm{B} 2)$. 


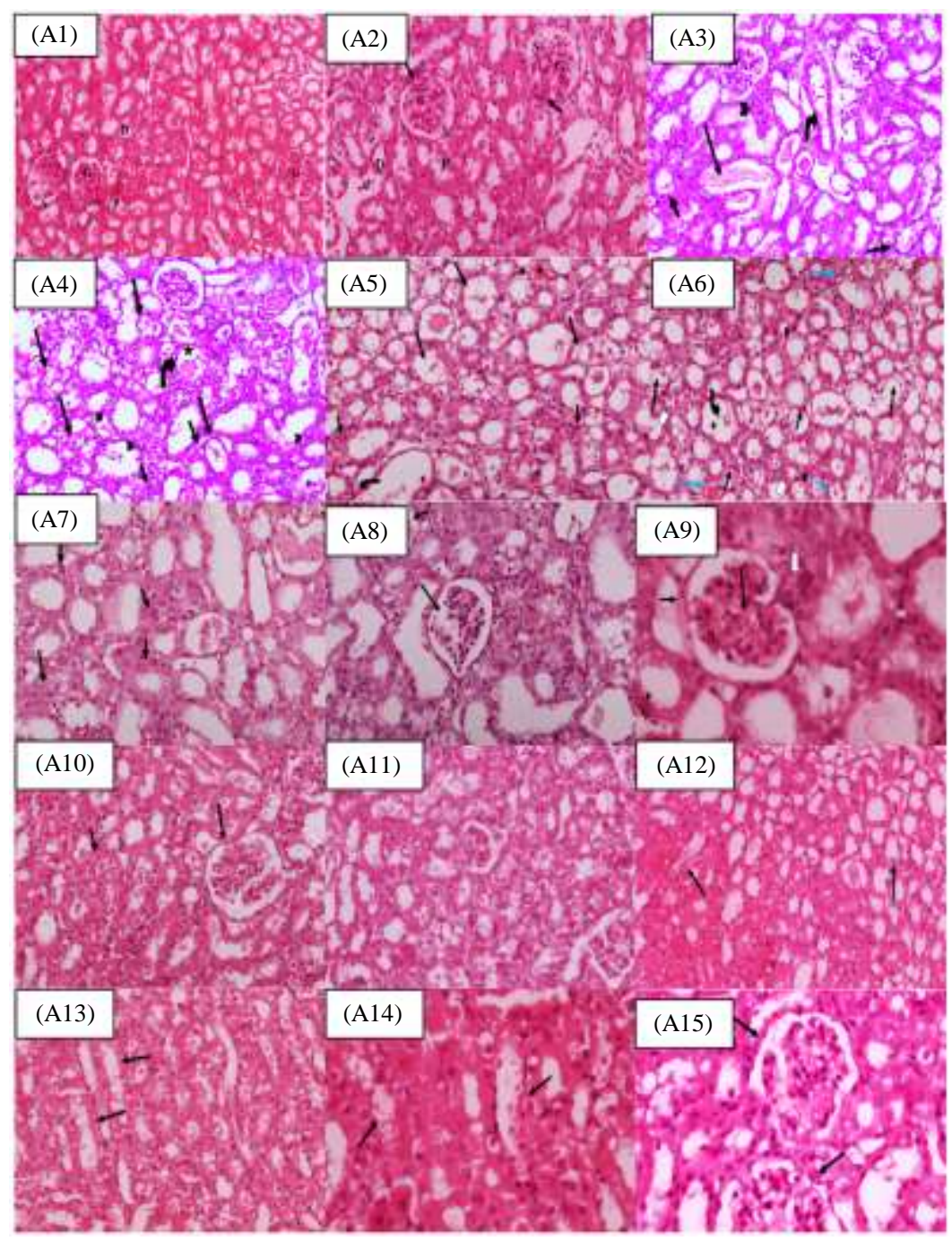

Fig. 2: Histopathological findings of the kidneys of the study groups using light microscopy H\&E (X 400). A1 and A2 represents photomicrograph of renal cortex from the control group (group I) and lactoferrin treated rat (group II) showing normal renal parenchyma; glomerulus $(\mathrm{G})$, proximal convoluted tubule $(\mathrm{P})$, distal convoluted tubules (D), Urinary (Bowman's) space (S) and vascular pole (arrow). A3-A9 represent histopathological findings of the kidneys of potassium dichromate-treated rats (group-III): A3: Photomicrograph of renal cortex showing intraluminal casts (long arrow), tubular degeneration (short arrow), dilated congested interstitial blood vessels (curved arrow), heamorragic hypercellular glomeruli (star) and inflammatory infiltrate (arrow head); A4: Photomicrograph of renal cortex showing tubular epethelial vaculation (long arrow), nuclear pyknosis (arrow head) and binucleation (short arrow) and widened Bowman's space (star) surrounding shrunken glomeruli (curved arrow); A5: Photomicrograph of renal cortex showing tubular epethilial vaculation (long arrow) and binucleation (short arrow), intraluminal esinophilic casts (curved arrow) and interstitial hemorrhage(star); A6: Photomicrograph of renal cortex showing tubular epethilial vaculation (long black arrow), intracytoplasmic hyaline droplets (open arrow), intraluminal casts (short blue arrow) pyknosis (arrow head) and binucleation (short black arrow), widened Bowman's space (star) surrounding atrophied glomeruli (curved arrow) and interstitial hemorrhage (long blue color);); A7: Photomicrograph of renal cortex showing tubular epithelial hyperplasia (long arrow) and karyomegally (short arrow); A8: Photomicrograph of renal cortex showing necrotic shrunken (short arrow) and lobulated glomeruli (long arrow). A9: Photomicrograph of renal cortex showing capillary congestion of glomerular tuft (long arrow), inflammatory cells around renal corpuscle (open arrow) and moderate thickening of the basement membrane of Bowman's capsule (short arrow). A10-A15 represent histopathological findings of the kidneys of potassium dichromate and lactoferrin co-treated rats (group-IV): A10: Photomicrograph of renal cortex showing mild glomerular damage (long arrow), with uniformly arranged regenerative renal tubules (short arrow); A11: Photomicrograph of renal cortex showing mild glomerular damage (long arrow) and mild damage of the tubular lining epithelium (short arrows); A12: Photomicrograph of renal cortex showing mild tubular damage, with granular damage of their lining epithelium (arrows); A13: Photomicrograph of renal cortex showing mild granular damage of the tubular lining epithelium (arrows); A14: Photomicrograph of renal cortex showing mild granular damage of the tubular lining epithelium (arrows); A15: Photomicrograph of renal cortex showing mild glomerular damage (arrows) 


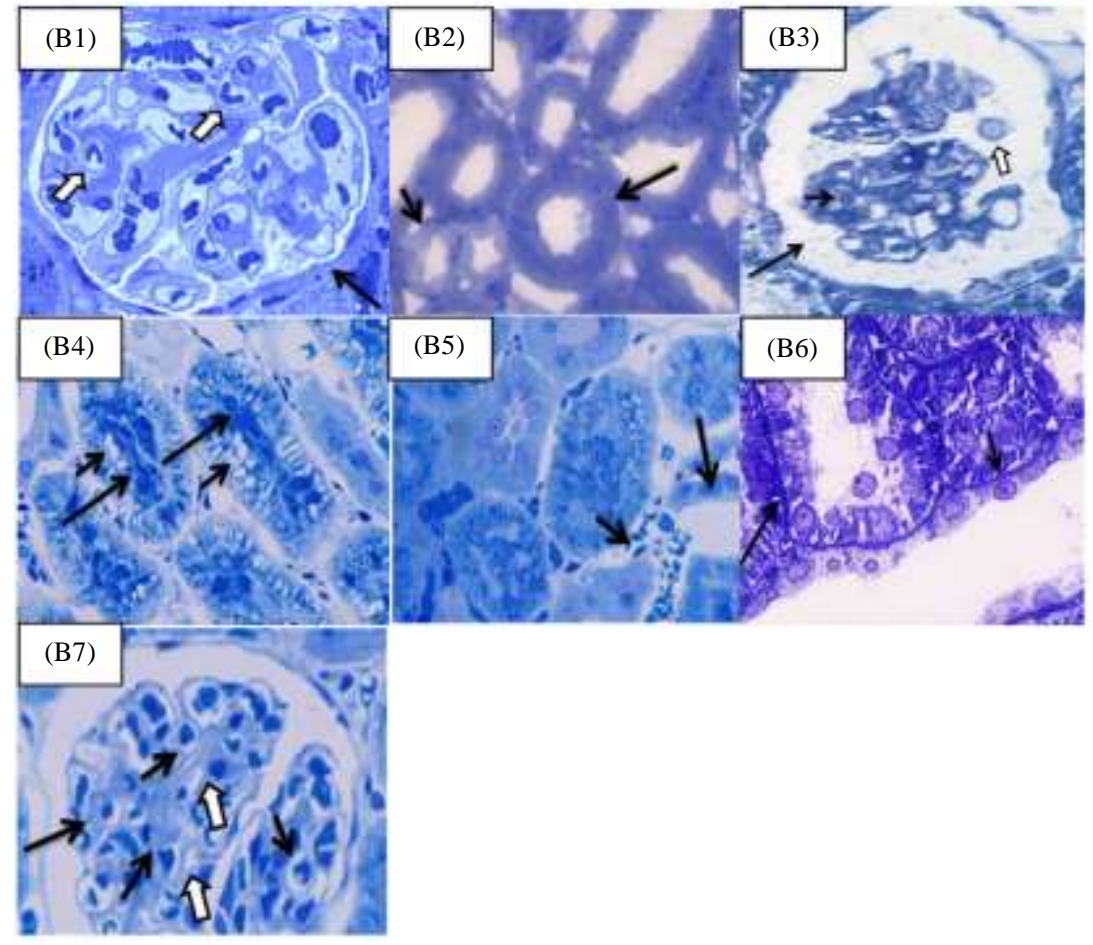

Fig. 3: Histopathological findings of the kidneys of the study groups using Semithin sections toluidine blue X1000. B1 and B2 represent photomicrographs of kidneys from the control group (group I) and lactoferrin treated rat (group II); B1: Photomicrograph showing normal appearance of glomerulus (long arrow), Bowman's space (short arrow) and mesangial cells (open arrow) of the control/Lf-treated rats; B2: Photomicrograph showing normal appearance of proximal tubule (long arrow) and distal tubule (short arrow). B3-B5 represent photomicrographs of the kidneys of potassium dichromatetreated rats (group-III): B3: Photomicrograph showing necrotic shrunken and lobulated glomerulus with widened Bowman's capsule (long arrow), some nuclei are dense (short arrow) while others appear pale (open arrow); B4: Photomicrograph showing proximal tubules with epithelial vaculations (short arrows) and intraluminal casts (long arrows); B5: Photomicrograph showing congestion of the blood vessels (long arrow) and interstitial inflammatory cells (short arrow). B6 and B7 represent photomicrographs of the kidneys of potassium dichromate and lactoferrin co-treated rats (group-IV): B6: Photomicrograph revealing PCT (long arrow) and DCT (short arrow) with more orless normal appearance; B7: Photomicrograph revealing more or less normal appearance of renal glomerulus with podocytes (long arrow), mesangial cells (short arrows) and capillaries (open arrow)

Table 1: Mean \pm SD of blood urea, serum creatinine, random blood glucose and total antioxidant capacity levels among the study groups

\begin{tabular}{llllll}
\hline & Group-I $(\mathrm{n}=10)$ & Group-II & Group-III & Group-IV \\
Biochemical parameters & Mean \pm SD & Mean \pm SD & Mean \pm SD & Mean \pm SD & P-value \\
\hline Blood urea $(\mathrm{mg} / \mathrm{dl})$ & $45.2 \pm 7.44^{\mathrm{a}, \mathrm{b}}$ & $38.8 \pm 7.49^{\mathrm{c}}$ & $69.4 \pm 44.15^{\mathrm{d}}$ & $45.2 \pm 4.02$ & $0.001^{*}$ \\
Serum creatinine $(\mathrm{mg} / \mathrm{dl})$ & $0.492 \pm 0.07^{\mathrm{a}, \mathrm{b}}$ & $0.402 \pm 0.03^{\mathrm{c}}$ & $0.566 \pm 0.13$ & $0.528 \pm 0.04$ & $0.001^{*}$ \\
Random blood glucose $(\mathrm{mg} / \mathrm{dl})$ & $113 \pm 15.23^{\mathrm{b}}$ & $110.8 \pm 27.95^{\mathrm{c}}$ & $140.8 \pm 50.27$ & $119 \pm 29.21$ & $0.021^{*}$ \\
Total antioxidant capacity $(\mathrm{mmol} / \mathrm{ml})$ & $8 \pm .0 .84^{\mathrm{b}}$ & $8.02 \pm 1.05^{\mathrm{c}}$ & $6.22 \pm 2.09^{\mathrm{d}}$ & $7.94 \pm 1.53$ & $0.001^{*}$ \\
\hline
\end{tabular}

* Statistically significant difference ( $<<0.05)$; Data presented in (mean \pm SD) using ANOVA test for comparison; Group-I: Control group; Group-II: Lactoferrin group; Group-III: Potassium dichromate group; Group-IV: Potassium dichromate + lactoferrin group; ${ }^{a}$ significant difference when comparing group-I Vs. group-II; ${ }^{b}$ significant difference when comparing group-I Vs. group-III; ${ }^{c}$ significant difference when comparing group-II Vs. group-III. ${ }^{d}$ significant difference when comparing group-III Vs. group-IV. No significance differences between group-I and IV

Using electron microscopy, the epithelial cells in a distal convoluted tubule of the control/Lf-treated rats revealed cells rest on the basement membrane and circumscribing the tubular lumen which is greatly reduced by the expanded blebs bulging into the lumen. Cells harbor a considerable number of elongated mitochondria with normal renal filtration barrier. Proximal tubule cells showed apical numerous microvilli (brush border), euochromatic rounded nucleus, some cytoplasmic vacuoles and mitochondria as presented in (Fig. 4C1-C5). 


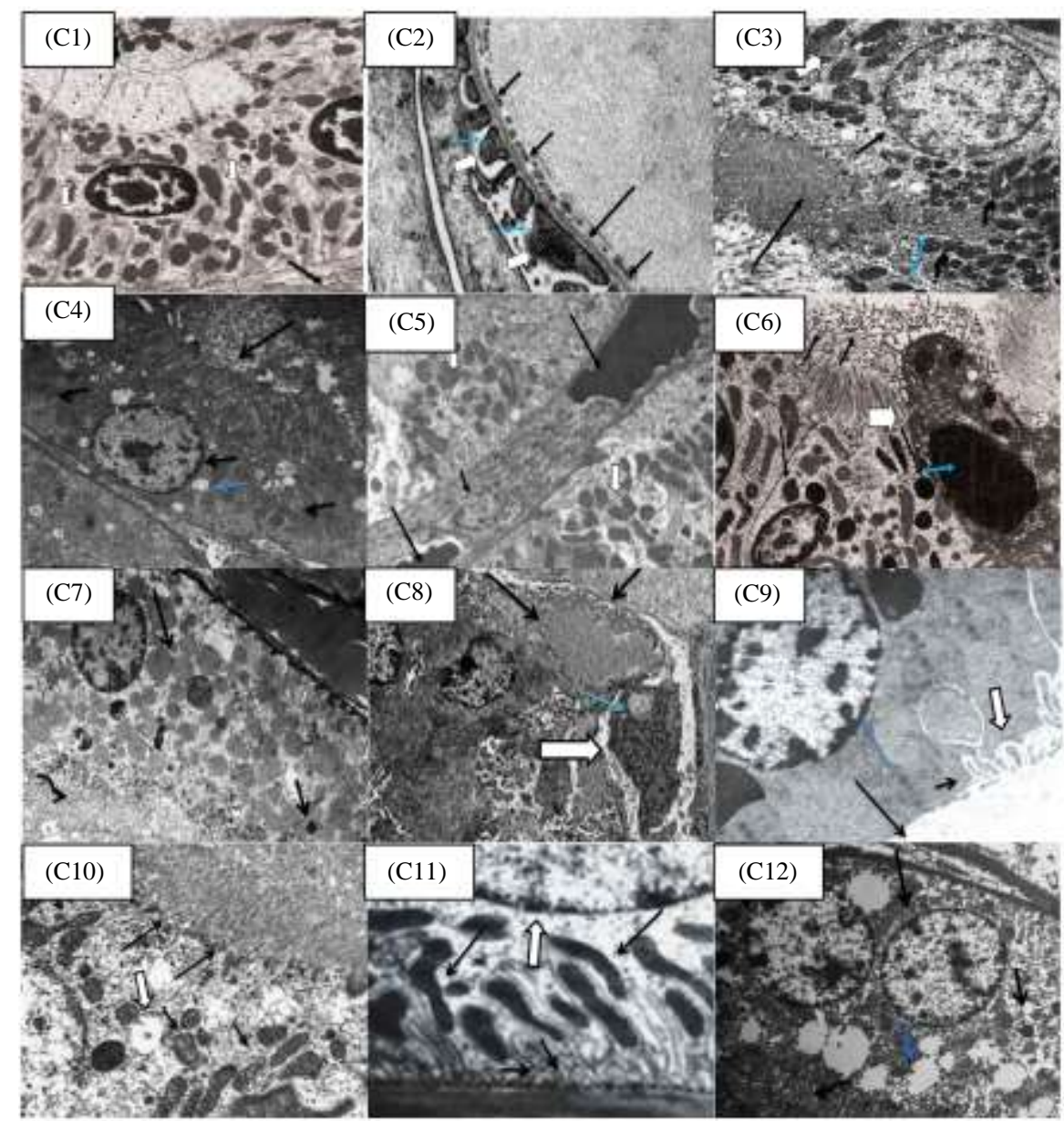

Fig. 4: Histopathological findings of the kidneys of the study groups using electron microscopy. C1-C5 represent photomicrographs of kidneys from the control group (group I) and lactoferrin treated rat (group II); C1: Epithelial cells in a distal convoluted tubule of the control/Lf-treated rats. Cells rest on the basement membrane (long arrow) and circumscribing the tubular lumen which is greatly reduced by the expanded blebs (short arrow) bulging into the lumen. Cells harbor a considerable number of elongated mitochondria (open arrow). (X 4300); C2: The kidney's filtration barrier appeared normal consisting of glomerular basement membranes (long arrow), fenestrated endothelium (short arrows) and secondary foot processes (open arrows) separated by filtration slits (blue arrows) (X 5200); C3: The proximal tubule cells show apical numerous microvilli (brush border) (long arrow), euochromatic rounded nucleus (short arrow), lysosomes (open arrow), RER (blue arrow) and basal membrane invaginations associated with elongated mitochondria (curved arrows). (X 5800); C4: The proximal tubule cells show apicalnumerous microvilli (brush border) (long arrow), euochromatic rounded nucleus (short arrow), some cytoplasmic vacuoles (blue arrow) and mitochondria (curved arrows). (X 58000); C5: Theinterstitial space between two tubules that contain blood capillaries (long arrow), interstitial cells (short arrow) and mitochondria (open arrow). (X4800). C6-C9 represent photomicrographs of the kidneys of potassium dichromate-treated rats (group-III): Theepithelial cells of the proximal tubule from the kidney cortex. Some mitochondria are swollen and many lysosomes (long arrows) are seen. Disintegrated microvilli (short arrows). A necrotic epithelial cell (open arrow) is seen with a pyknotic nucleus (blue arrow) (X 5000); C7: The epithelial cells of the proximal tubule from the kidney cortex. Some mitochondria are swollen (long arrows) and many lysosomes (short arrows) are seen. Disintegrated microvilli are demonstrated in the lumen (curved aarrow). (X 5800); C8: The congested glomerular capillaries (long arrow) with irregularly thickened capillary basement membranes (short arrow), damaged podocytes (open arrow) having large intraluminal vacuoles (blue arrow) (X 4800); C9: Apparent increase in thickness of the basal lamina (long arrow) with partial loss of basal infoldings (short arrow). Mitochondria (open arrow) and condensed relatively dense nucleus (blue arrow). (X 4000).C10-C12 represent photomicrographs of the kidneys of potassium dichromate and lactoferrin co-treated rats (group-IV); C10: The proximal convoluted tubule cell showed restoration of the integrity of the apical brush border (long arrows), mitochondria more or less normal in shape and size (short arrows) and a few vacuoles (open arrow) are seen (X 4800); C11: Multiple basal mitochondria (long arrows) and restored basal infoldings (short arrows) and nucleus (open arrow). (X 4000); C12: The proximal tubule in the renal cortex showednormal ovoid nucleus (long arrow), mitochondria appeared rather normal in shape (short arrow), with little restored regular apical brush border (curved arrow), some vaculations are still seen (blue arrow). (X 5800) 
As regards the histopathological findings of the kidneys of potassium dichromate-treated rats (group-III), light microscopic examination revealed intraluminal casts, tubular degeneration and dilation, dilated congested interstitial blood vessels, hypercellular glomeruli with hemorrhage and interstitial inflammatory infiltrate with hemorrhagic blood masses of the renal cortex with degenerations in the tubular epethelial cells. Widened Bowman's space was surrounded atrophied and shrunken glomeruli (Fig. 2A3-A9 and Fig. 3B3-B5). An electron micrograph showed congested glomerular capillaries with irregularly thickened capillary basement membranes, damaged podocytes with large intraluminal vacuoles as presented in (Fig. 4C6-C9).

Regarding to the histopathologic effects from adding lactoferin co-therapy to PDC (group-IV), H\& E and semithin toluidine blue sections revealed decreased glomerular damage with regeneration of the renal tubules with more or less normal podocytes, PCT and DCT (Fig. 2A10-A15 and 3B6-B7). Electron micrograph of the kidney showed restoration of the integrity of the apical brush border, mitochondria more or less normal in shape and size and a few vacuoles in the proximal convoluted tubular cells with mitochondria appeared rather normal in shape as presented in (Fig. 4C10-C12).

\section{Discussion}

The liver and kidney are the most important organs for the metabolism, detoxification, storage and excretion of xenobiotics and their metabolites and is particularly susceptible to multi-agent harm (Park et al., 2014). The kidney is the target organ of systemically absorbed chromate and in acute chromium exposure, nephrotoxicity or total renal shutdown may be the primary cause of death. The chromate's tubular damage and nephrotoxic effect resulted from its accumulation in vacuoles within the proximal tubular cells, resulting in slower excretion and long-term remaining of $\mathrm{Cr}$ in the kidney (Hegazy et al., 2016).

In this research, the adverse pathological effects of PDC on the kidneys of male rats and the beneficial effects of co-treatment with LF were evaluated.

In the current study, single dose PDC injection for 14 days (group-III) induced acute kidney injury in rats, as evidenced by significant renal function test alterations, confirmed by severe changes in PDC group histopathology, especially tubular necrosis. In the current research, the assessment of kidney functions was evaluated by estimating the levels of blood urea and serum creatinine. Compared to other groups, there was a statistically significant increase in levels of urea and creatinine in the PDC-treated group. This was in line with proven literature evidence of alteration of renal functions caused by $\mathrm{Cr}$. The rise in these parameters was due to loss of functional integrity in the kidney and renal tubule distortion as a consequence of $\mathrm{Cr}$ administration (Venter et al., 2017). Many studies were in line with our findings and clarified that $\mathrm{Cr}$-induced renal dysfunction could be due to $\mathrm{Cr}$ renal tubular damage and cell debris obstruction (Sahu et al., 2014; Hegazy et al., 2016).

In the current research, it was evident that, given the acute increase in blood glucose in the PDC treated group (group-III), the occurrence of renal function disorder was also recorded, our findings were in line with (El-Guendouz et al., 2020) results. Arreola-Mendoza et al., (2006) however, found that blood glucose in the PDC treated rats was not significantly elevated, though apparent glucosuria was reported and this was explained by the lack of absorption due to proximal tubular injury caused by this metal. The surprisingly toxic aspect of urea was illustrated by a study by Koppe and his colleagues. They proposed that urea is directly responsible for the impaired secretion of insulin in chronic kidney disease and a specific protein named phosphofruktokinase-I was found in the pancreatic beta cells in their research. The function of this protein was modified by an increase in urea in the blood that occurred during kidney disease. Increased urea causes insulin release from the beta cells of the pancreas to be impaired. This induces oxidative stress and excessive phosphofructokinase-I glycosylation, which creates an imbalance of blood glucose and may contribute to diabetes (Koppe et al., 2016).

The kidney is the main route of excretion of $\mathrm{Cr}$ and acute exposure to PDC in rats has been reported to cause an increase in the content of $\mathrm{Cr}$ in the kidney. While $\mathrm{Cr}$ itself does not produce free radicals directly, it indirectly creates various radicals such as superoxide, peroxynitrite, nitric oxide and hydroxyl that cause damage consistent with oxidative stress (Mehany et al., 2013). Since the nephrotoxic effect of PDC is mainly due to oxidative stress caused by it, the present study evaluated TAO levels in the groups studied and showed a significant decrease in TAO levels in the serum of the PDC group (group-III). Such reports were in line with many investigators (Hegazy et al., 2016; El-Guendouz et al., 2020).

With regard to the effect of PDC on proximal convoluted tubules, tubular degeneration, dilation, intraluminal casting, nuclear pycnosis and binucleation and vacuolar formation of proximal convoluted tubules were shown in the present research. Similar findings were previously demonstrated by Hegazy et al., who revealed significant necrobiotic changes in almost proximal convoluted tubules and clarified that chromate's tubular damage and nephrotoxic effect resulted from its accumulation in vacuoles within the proximal tubular cells, leading to slow excretion and long-term retention of $\mathrm{Cr}$ in the kidney (Hegazy et al., 2016). They also stated that the treated animals showed intraluminal and intracytoplamic accumulation of acidophilic hyaline and renal cast content (Hegazy et al., 2016). Also other studies 
showed similar findings (El-Mahalaway et al., 2015; Hanan et al., 2019).

The key intracellular source of ROS is mitochondria and they have a very efficient antioxidant system. The targets of metal toxicity are mitochondria. Oxidative stress results in dysfunction of the mitochondria and apoptosis (García-Niño et al.,2013). In the current research, electron microscopic analysis of group-III proximal and distal convoluted tubular cells showed a shrunken nucleus with chromatin margination (a sign of cell apoptosis). These results were in line with those of prior research (Abdel-Moneim and Said, 2007; Rashedy et al., 2013; Morya and Vachhrajani, 2014; El-Mahalaway et al., 2015).

To prevent the toxicities caused by chemicals, many natural products have been used to protect against such toxicities (Guo, 2017). Lactoferrin (LF, formerly known as lactotransferrin) is an iron-binding glycoprotein, belonging to the transferrin protein family, together with serum Transferrin (sTf), Ovotransferrin (Otrf), melanotransferrin and the inhibitor of carbonic anhydrase (González-Chávez et al., 2009). High levels of LF-mRNA and protein were found in the kidneys during screening for LF expression in different organs. This showed that LF is provided by the kidneys and that LF may have important functions in this organ's intrinsic immunity as well as in the antioxidant and other kidney safety systems against any other nonmicrobial injuries, such as ischemia-reperfusion and inflammation (Åbrink et al., 2010).

The current study also showed that the serum urea and creatinine levels in the LF-treated group (group-II) were statistically significantly reduced compared to the control group (group-I), in line with the reported vital function of LF for kidney health (Åbrink et al., 2010). However; there was no statistical difference between control group (groupI) and PDC and LF-treated group (group-IV). Serum creatinine and urea levels were decreased in PDC and LF co-treated group (group-IV) denoting the importance of LF in restoration of kidney function induced by $\mathrm{Cr}$ nephrotoxicity. LF suppressed oxidative stress-induced cell death and apoptosis in human kidney tubular epithelial cells, the latest study findings found. In addition, by suppressing expression of the profibrogenic genes CTGF, PAI-1 and collagen I, lactoferrin inhibited TGF- $\beta 1$-induced renal fibrosis (Hsu et al., 2020).

In the current research, LF (group-IV) treatment of rats significantly protected the kidney against oxidative stress caused by PDC, as evidenced by the preservation of normal TAO. In line with this, (Kimoto et al., 2013) showed a protective effect of LF in rats against cisplatininduced nephrotoxicity. In addition, several studies have recorded the antioxidant effect of LF and suggested that the binding of LF to cells limits the process of membrane lipid peroxidation, since LF is not entirely saturated and is capable of removing free iron radicals that are cytotoxic activators of lipid peroxidation and oxidative stress, thereby suppressing free radical harm (Latorre et al., 2010; Ogasawara et al., 2014; Hessin et al., 2015; Hegazy et al., 2016).

The light microscopic photomicrographs obtained from the PDC+LF treated rats (group-IV) showed relative restoration of the normal renal architecture and reversion of most of the destructive changes showing mild glomerular damage, mild tubular damage, granular damage with uniformly organised regenerative renal tubules of their lining epithelium. These findings provide further evidence of the protective effect of LF against nephrotoxicity caused by metals in rats, in agreement with several investigators (Latorre et al., 2010; Kimoto et al., 2013; Ogasawara et al., 2014; Hessin et al., 2015; Hegazy et al., 2016).

In addition, group-IV showed an increase in PDCinduced ultrastructural improvements, as evidenced by electron microscopic examination. The presence of mitotic tubular cell behaviour was indicative of regeneration of the tubular cells. In addition, the integrity of brush boundary, mitochondria and basal infolding were restored. In humans and laboratory animals, it has been documented that LF has a protective effect against several toxicants. It increases the resistance of the body to several harmful factors and protects tissues from damage, as well as decreases chromosomal aberrations caused by certain chemicals (Hsu et al., 2020).

\section{Conclusion}

The current study provides evidence of LF's renoprotective role against PDC-induced renal damage by increasing antioxidant activity to restore chromium exposure-induced functional and structural renal damage.

\section{Study Limitation}

Lack of using multi-dose design (low, medium and high dose) of lactoferrin to evaluate the possible doseeffect relationship was the main limitation of the current study which could be designed in future researches.

\section{Funding}

The current research was funded partially by South Valley University, Faculty of Medicine, Qena, Egypt.

\section{Authors' Contributions}

Mohammed H. Hassan: Study concept and design, experimental procedures, blood sampling and biochemical assays, statistical analysis, literature research, first manuscript drafting.

Dorreia Abd-Alla Mohamed Zaghloul: Study concept and design, experimental procedures, histopathological examinations, statistical analysis. 
Marwa Ahmed Mahmoud and Rana Toghan: Experimental procedures, histopathological examinations, literature research.

Zamzam Nasrallah Abdel-Moaty: Experimental procedures, histopathological examinations, literature research, statistical analysis.

\section{Ethics Approval}

All experimental protocols were performed in accordance with the local institutional guidelines and approved by the Animal Ethical Committee, South Valley University-Qena, Egypt.

\section{References}

Abdel-Moneim, A. M., \& Said, K. M. (2007). Acute effect of cadmium treatment on the kidney of rats: biochemical and ultrastructural studies. Pakistan Journal of Biological Sciences: PJBS, 10(20), 3497-3506.

https://doi.org/10.3923/pjbs.2007.3497.3506

Åbrink, M., Larsson, E., Gobl, A., \& Hellman, L. (2000). Expression of lactoferrin in the kidney: implications for innate immunity and iron metabolism. Kidney International, 57(5), 2004-2010. https://doi.org/10.1046/j.1523-1755.2000.00050.x

Adlerova, L., Bartoskova, A., \& Faldyna, M. (2008). Lactoferrin: a review. Veterinarni Medicina, 53(9), 457-468. https://doi.org/10.17221/1978-VETMED

Al-Othman, Z. A., Ali, R., \& Naushad, M. (2012). Hexavalent chromium removal from aqueous medium by activated carbon prepared from peanut shell: adsorption kinetics, equilibrium and thermodynamic studies. Chemical Engineering Journal, 184, 238-247. https://doi.org/10.1016/j.cej.2012.01.048

Arreola-Mendoza, L., Reyes, J. L., Melendez, E., Martín, D., Namorado, M. C., Sanchez, E., \& Del Razo, L. M. (2006). Alpha-tocopherol protects against the renal damage caused by potassium dichromate. Toxicology, 218(2-3), 237-246. https://doi.org/10.1016/j.tox.2005.11.010

Ayache, J., Beaunier, L., Boumendil, J., Ehret, G., \& Laub, D. (2010). Sample preparation handbook for transmission electron microscopy: techniques (Vol. 2). Springer Science \& Business Media. https://doi.org/10.1007/978-1-4419-5975-1

Calvello, R., Aresta, A., Trapani, A., Zambonin, C., Cianciulli, A., Salvatore, R., ... \& Panaro, M. A. (2016). Bovine and soybean milk bioactive compounds: Effects on inflammatory response of human intestinal Caco- 2 cells. Food Chemistry, 210, 276-285.

https://doi.org/10.1016/j.foodchem.2016.04.067
El-Guendouz, S., Zizi, S., Elamine, Y., \& Lyoussi, B. (2020). Preliminary screening of the possible protective effect of Moroccan propolis against chromium-induced nephrotoxicity in animal model. Veterinary $\quad$ World, 1327. https://doi.org/10.14202/vetworld.2020.1327-1333

El-Mahalaway, A. M., Salem, M. M., \& Mousa, A. M. (2015). The effect of potassium dichromate on convoluted tubules of the kidney of adult male albino rats and the possible protective role of ginseng: a histological and immunohistochemical study. Egyptian Journal of Histology, 38(2), 157-167. https://doi.org/10.1097/01.EHX.0000464738.41270.06

El-Saad, A. M. A., Abdel-Moneim, A. M., \& AbdelKarim, H. M. (2010). N-acetylcysteine an Allium plant compound protects against chromium (VI) induced oxidant stress and ultrastructural changes of pancreatic beta-cells in rats. Journal of Medicinal Plants Research, 4(21), 2290-2297. https://academicjournals.org/journal/JMPR/articleabstract/2EECBD417269

Gabe, M. (1976). Histochemical techniques. Masson, Paris New York Barcelona Milan and Springer, New York Heidelberg Berlin.

Gamble, M. (2008). The hematoxylins and eosin. In: Bancroft JD, Gamble M, editors. Theory and practice of histological techniques. 6th ed. London (UK): Churchill Livingston/Elsevier Inc. 121-134. https://doi.org/10.1016/B978-0-443-10279-0.50016-6

García-Niño, W. R., Tapia, E., Zazueta, C., ZatarainBarrón, Z. L., Hernández-Pando, R., Vega-García, C. C., \& Pedraza-Chaverrí, J. (2013). Curcumin pretreatment prevents potassium dichromate-induced hepatotoxicity, oxidative stress, decreased respiratory complex I activity and membrane permeability transition pore opening. EvidenceBased Complementary and Alternative Medicine, 2013. https://doi.org/10.1155/2013/424692

González-Chávez, S. A., Arévalo-Gallegos, S., \& Rascón-Cruz, Q. (2009). Lactoferrin: structure, function and applications. International Journal of Antimicrobial Agents, 33(4), 301-e1. https://doi.org/10.1016/j.ijantimicag.2008.07.020

Guo, Z. (2017). The modification of natural products for medical use. Acta Pharmaceutica Sinica B, $7(2)$,

119-136. https://doi.org/10.1016/j.apsb.2016.06.003

Hanan, E. M., Alshymaa, O. A., Samaa, S., \& Helmy, H. O. (2019). Structural Changes Induced by Potassium Dichromate in Renal Cortex of Adult Male Albino Rats and the Possible Protective Role of Selenium. The Medical Journal of Cairo University, 87(March), 661-675. https://doi.org/10.21608/mjcu.2019.52521 
Hassan, M. H., Awadalla, E. A., Ali, R. A., Fouad, S. S., \& Abdel-Kahaar, E. (2020). Thiamine deficiency and oxidative stress induced by prolonged metronidazole therapy can explain its side effects of neurotoxicity and infertility in experimental animals: Effect of grapefruit co-therapy. Human \& Experimental Toxicology, 39(6), 834-847. https://doi.org/10.1177/0960327119867755

Hassan, M. H., Desoky, T., Sakhr, H. M., Gabra, R. H., \& Bakri, A. H. (2019). Possible metabolic alterations among autistic male children: clinical and biochemical approaches. Journal of Molecular Neuroscience, 67(2), 204-216. https://doi.org/10.1007/s12031-018-1225-9

Hegazy, R., Salama, A., Mansour, D., \& Hassan, A. (2016). Renoprotective effect of lactoferrin against chromium-induced acute kidney injury in rats: involvement of IL-18 and IGF-1 inhibition. PloS One, 11(3), e0151486. https://doi.org/10.1371/journal.pone.0151486

Hessin, A., Hegazy, R., Hassan, A., Yassin, N., \& Kenawy, S. (2015). Lactoferrin enhanced apoptosis and protected against thioacetamide-induced liver fibrosis in rats. Open access Macedonian Journal of Medical Sciences, 3(2), 195. https://doi.org/10.3889/oamjms.2015.038

Hsu, Y. H., Chiu, I. J., Lin, Y. F., Chen, Y. J., Lee, Y. H., \& Chiu, H. W. (2020). Lactoferrin Contributes a Renoprotective Effect in Acute Kidney Injury and Early Renal Fibrosis. Pharmaceutics, 12(5), 434. https://doi.org/10.3390/pharmaceutics 12050434

Kart, A., Koc, E., Dalginli, K. Y., Gulmez, C., Sertcelik, M., \& Atakisi, O. (2016). The therapeutic role of glutathione in oxidative stress and oxidative DNA damage caused by hexavalent chromium. Biological Trace Element Research, 174(2), 387-391. https://doi.org/10.1007/s12011-016-0733-0

Kimoto, Y., Nishinohara, M., Sugiyama, A., Haruna, A., \& Takeuchi, T. (2013). Protective effect of lactoferrin on Cisplatin-induced nephrotoxicity in rats. Journal of Veterinary Medical Science, 75(2), 159-164. https://doi.org/10.1292/jvms.12-0154

Koppe, L., Nyam, E., Vivot, K., Fox, J. E. M., Dai, X. Q., Nguyen, B. N., ... \& Poitout, V. (2016). Urea impairs $\beta$ cell glycolysis and insulin secretion in chronic kidney disease. The Journal of Clinical Investigation, 126(9), 3598-3612. https://doi.org/10.1172/JCI86181

Latorre, D., Puddu, P., Valenti, P., \& Gessani, S. (2010). Reciprocal interactions between lactoferrin and bacterial endotoxins and their role in the regulation of the immune response. Toxins, 2(1), 54-68. https://doi.org/10.3390/toxins2010054
Mehany, H. A., Abo-youssef, A. M., Ahmed, L. A., Arafa, E. S. A., \& Abd El-Latif, H. A. (2013). Protective effect of vitamin $\mathrm{E}$ and atorvastatin against potassium dichromate-induced nephrotoxicity in rats. Beni-Suef University Journal of Basic and Applied Sciences, 2(2), 96-102. https://doi.org/10.1016/j.bjbas.2013.02.002

Mishra, S., \& Bharagava, R. N. (2016). Toxic and genotoxic effects of hexavalent chromium in environment and its bioremediation strategies. Journal of Environmental Science and Health, Part C, 34(1), $1-32$. https://doi.org/10.1080/10590501.2015.1096883

Morya, K., \& Vachhrajani, K. D. (2014). Impairment of renal structure and function following heterogeneous chemical mixture exposure in rats. Indian Journal of Experimental Biology, 52(4), 332-43. http://nopr.niscair.res.in/handle/123456789/27968

Navya, K., Chandrasekhar, Y., \& Anilakumar, K. R. (2018). Evaluation of potassium dichromate (K 2 $\mathrm{Cr} 2 \mathrm{O}$ 7)-induced liver oxidative stress and ameliorative effect of Picrorhiza kurroa extract in Wistar albino rats. Biological Trace Element Research, 184(1), 154-164. https://doi.org/10.1007/s12011-017-1172-2

Ogasawara, Y., Imase, M., Oda, H., Wakabayashi, H., \& Ishii, K. (2014). Lactoferrin directly scavenges hydroxyl radicals and undergoes oxidative selfdegradation: a possible role in protection against oxidative DNA damage. International Journal of Molecular Sciences, 15(1), 1003-1013. https://doi.org/10.3390/ijms15011003

Park, Y. C., Lee, S., \& Cho, M. H. (2014). The Simplest Flowchart Stating the Mechanisms for Organic Xenobiotics-induced Toxicity: Can it Possibly be Accepted as a "Central Dogma" for Toxic Mechanisms?. Toxicological Research, 30(3), 179-184. https://doi.org/10.5487/TR.2014.30.3.179

Rashedy, A. H., Solimany, A. A., Ismail, A. K., Wahdan, M. H., \& Saban, K. A. (2013). Histopathological and functional effects of antimony on the renal cortex of growing albino rat. International Journal of Clinical and Experimental Pathology, 6(8), 1467. https://www.ncbi.nlm.nih.gov/pmc/articles/PMC372 6962/

Sahu, B. D., Koneru, M., Bijargi, S. R., Kota, A., \& Sistla, R. (2014). Chromium-induced nephrotoxicity and ameliorative effect of carvedilol in rats: Involvement of oxidative stress, apoptosis and inflammation. Chemico-Biological Interactions, 223, 69-79. https://doi.org/10.1016/j.cbi.2014.09.009 
Saleem, T. H., Abo El-Maali, N., Hassan, M. H., Mohamed, N. A., Mostafa, N. A., Abdel-Kahaar, E., \& Tammam, A. S. (2018). Comparative protective effects of $\mathrm{N}$-acetylcysteine, $\mathrm{N}$-acetyl methionine and $\mathrm{N}$-acetyl glucosamine against paracetamol and phenacetin therapeutic doses-induced hepatotoxicity in rats. International journal of Hepatology, 2018. https://doi.org/10.1155/2018/7603437

Saleem, T. H., Shehata, G. A., Toghan, R., Sakhr, H. M., Bakri, A. H., Desoky, T., ... \& Hassan, M. H. (2020). Assessments of amino acids, ammonia and oxidative stress among cohort of egyptian autistic children: correlations with electroencephalogram and disease severity. Neuropsychiatric Disease and Treatment, 16, 11. https://doi.org/10.2147/NDT.S233105

Sun, H., Brocato, J., \& Costa, M. (2015). Oral chromium exposure and toxicity. Current Environmental Health Reports, 2(3), 295-303. https://doi.org/10.1007/s40572-015-0054-z

Teklay, A. (2016.) Physiological effect of chromium exposure: A review. International Journal of Food Science, Nutrition and Dietetics, S7,001, 1-11. https://doi.org/10.19070/2326-3350-SI07001
Venter, C., Oberholzer, H. M., Cummings, F. R., \& Bester, M. J. (2017). Effects of metals cadmium and chromium alone and in combination on the liver and kidney tissue of male Spraque-Dawley rats: An ultrastructural and electron-energy-loss spectroscopy investigation. Microscopy Research and Technique, 80(8), 878-888. https://doi.org/10.1002/jemt.22877

Wu, F., Sun, H., Kluz, T., Clancy, H. A., Kiok, K., \& Costa, M. (2012). Epigallocatechin-3-gallate (EGCG) protects against chromate-induced toxicity in vitro. Toxicology and Applied Pharmacology, 258(2), $166-175$ https://doi.org/10.1016/j.taap.2011.10.018

Yao, X., Bunt, C., Cornish, J., Quek, S. Y., \& Wen, J. (2013). Improved RP-HPLC method for determination of bovine lactoferrin and its proteolytic degradation in simulated gastrointestinal fluids. Biomedical Chromatography, 27(2), 197-202. https://doi.org/10.1002/bmc.2771 